msh-mss Mathématiques et sciences humaines

145 | Printemps 1999

Géométrie et vision

\title{
In mémoriam : Germain Kreweras
}

In mémoriam: Germain Kreweras

Marc Barbut

\section{OpenEdition}

Journals

Édition électronique

URL : http://journals.openedition.org/msh/2810

DOI : $10.4000 / \mathrm{msh} .2810$

ISSN : 1950-6821

\section{Éditeur}

Centre d'analyse et de mathématique sociales de l'EHESS

\section{Édition imprimée}

Date de publication : 1 mars 1999

ISSN : 0987-6936

\section{Référence électronique}

Marc Barbut, «In mémoriam : Germain Kreweras », Mathématiques et sciences humaines [En ligne], 145 | Printemps 1999, mis en ligne le 10 février 2006, consulté le 23 juillet 2020. URL : http:// journals.openedition.org/msh/2810; DOI : https://doi.org/10.4000/msh.2810 


\section{Germain KREWERAS}

Il y a un an, au début du mois de mars 1998, disparaissait Germain KREWERAS, Professeur émérite de Mathématiques à l'Université Pierre et Marie Curie (Paris VI), où il enseigna longtemps la Combinatoire dans le cadre de l'Institut de Statistique des Universités de Paris (I.S.U.P.).

En lui, Mathématiques, Informatique et Sciences humaines perdait l'un de ses amis les plus anciens et les plus fidèles, qui fut aussi un collaborateur particulièrement actif et efficace de la revue.

Membre de notre Comité de lecture depuis la création de la revue en 1962, il a participé à quasiment toutes les réunions de cette instance. Il ne manquait pas d'y faire des suggestions, d'y donner des avis, toujours pertinents, et généralement suivis.

Jamais il n'a rechigné à la tâche, combien ingrate parfois, de lire, critiquer, corriger, amender souvent, refuser parfois, des articles soumis à la revue et relevant des domaines dont il était un spécialiste reconnu : analyse combinatoire, ensembles ordonnés discrets, praxéologie mathématique.

De ses compétences et des questions auxquelles il s'est intéressé en Mathématiques discrètes, qu'elles soient "pures" ou "appliquées", les deux listes que l'on peut lire ci-après, permettent de se faire une idée convenable.

D'abord, la liste des dix principaux articles dont, au fil des ans, il a donné la primeur à notre revue; pour lui, d'ailleurs, c'était aussi sa revue.

Ensuite, celle de quelques "mots-clés" que l'on a extrait de ces textes en distinguant les mots mathématiques de ceux qui se rapportent aux Sciences Sociales.

C'est en 1956 que j'ai fait la connaissance de Germain Kreweras, lors des séances de travail hebdomadaires qui avaient lieu le samedi matin, 2 rue Chauchat, puis dans le même quartier, et tout près des "Folies Bergères", au 17 rue Richer. Elles réunissaient autour de Georges-Th. Guilbaud, les principaux collaborateurs du Bureau Universitaire de Recherche Opérationnelle de l'I.S.U.P. : outre Germain Kreweras, Jean Bouzitat, Georges Morlat, Edouard Valette, Micheline Petruszewycz et quelques autres. C'étaient des heures d'intense "brain-storming", dans une ambiance néanmoins très détendue, à propos de problèmes posés par la modélisation de la gestion des stocks, ou celle des files d'attente ou encore de programmation linéaire ; et, bien entendu et surtout, de Mathématiques. Germain Kreweras y passait très fréquemment à la "planche" ; il y fut particulièrement fécond, et enrichissant pour tous.

C'est vers la même époque, fin des années 50, début des années 60, et également au 17 de la rue Richer, que Germain Kreweras (il dirigeait alors le Centre de Recherches Économiques Appliquées, bureau d'études qui dépendait, je crois, de la Caisse des Dépôts et Consignations) participait assidument aux activités d'un petit groupe amical, dont j'étais le secrétaire : la Commission de Terminologie de la Société Française de Recherche 
Opérationnelle, commission dont deux autres "piliers" furent François Le Lionnais et le R.P. Dominique Dubarle.

Les travaux de cette commission, consistant en des définitions de termes, mathématiques ou non, de la Recherche Opérationnelle, furent publiés en feuilleton plusieurs années de suite dans la Revue Française de Recherche Opérationnelle. Germain Kreweras, passionné de terminologie, fut l'auteur de nombre des articles retenus.

Mais sa culture n'était pas que mathématique. Ce français originaire de la Lithuanie avait la maîtrise de plusieurs langues étrangères dont l'allemand et, bien sûr, le russe, mais aussi le grec et le latin. Profondément pétri d'humanité et d'une culture "classique" très étendue, ce fin lettré était d'ailleurs tout le contraire d'un cuistre ou d'un pédant. Aussi estce avec une parfaite simplicité qu'il avouait son goût pour les films burlesques, ceux des Marx Borthers notamment.

Ses propos toujours limpides alliaient avec bonheur, simplicité, humour et souvent ironie ; mais ironie gentille, et non féroce.

Ajouterai-je qu'ayant au plus haut point le sens des réalités, et que sa tête bien faite reposant sur des pieds bien solides, il était aussi tout le contraire d'un songe-creux ?

En définitive, Germain Kreweras fut, au meilleur sens du terme, un honnête homme dans ce siècle.

Marc BARBUT

\section{BIBLIOGRAPHIE DE GERMAIN KREWERAS DANS LA REVUE MATHÉMATIQUES, INFORMATIQUE ET SCIENCES HUMAINES}

[1] KREWERAS, G., "Les décisions collectives", Math. Sci. hum., n², 1963, 25-35.

[2] KREWERAS, G., "Une dualité élémentaire souvent utile dans les problèmes combinatoires", Math. Sci. hum., n³, 1963, 31-41.

[3] KREWERAS, G., "Dénombrements systématiques de relations binaires externes", Math. Sci. hum., n²6, 1969, 5-15.

[4] KREWERAS, G., "Intermédiarité, dendroïdes et quasi-dualité", Math. Sci. hum., $\mathrm{n}^{\circ} 29,1970,5-15$.

[5] KREWERAS, G., "Dénombrements relatifs à une définition combinatoire de la continuité sur un graphe", Math. Sci. hum., n51, 1975, 13-24.

[6] KREWERAS, G., "Les préordres totaux compatibles avec un ordre partiel", Math. Sci. hum., n53, 1976, 5-30.

[7] KREWERAS, G., "Polynômes de Stanley et extensions linéaires d'un ordre partiel", Math. Sci. hum., n73, 1981, 97-116.

[8] KREWERAS, G., "Approche bayésienne des phénomènes paranormaux. (Contribution à une analyse de la notion de croyance)", Math. Sci. hum., n81, 1983, 5966.

[9] KREWERAS, G., "Sur quelques problèmes relatifs au vote pondéré", Math. Sci. hum., n84, 1983, 45-63.

[10] KREWERAS, G., "Recouvrements d'un rectangle de largeur 3 à l'aide de triminos", Math. Inf. Sci. hum., n¹30, 1995, 27-31. 
Mathématiques

Arbres

Clique

Continuité sur un graphe

Décrochement

Dendroïdes

Différence n-ième d'un produit

Extension linéaire

Forme spectrale

Graphe biparti

Homomorphisme strict

h-partage

Idéaux

Inversion de Mobius

matrice d'incidence

Maximaux - minimaux

Nombres d'Euler

Nombres de Narayama

Ordres alternés

Ordre en épi

Ordres homogènes

Parties - partitions

Permutations "up-down"

Polynôme de Stanley

Préordre complet

Produit d'ordres totaux

Suite de Fibonacci

Suite de Young

Tassement et gonflement

Théorème de Bayes

Treillis des partitions

Triangle de Pascal

Triangle de Stirling

Triminos

\section{Sciences sociales}

Analyse de la notion de croyance

Cohérence

Conviction

Décisions collective

Degré de certitude

Degré de croyance

Effet Condorcet

Eloignement

Ensemble totalement désordonné

Incomparabilité

Indifférence

Intérêt général

Intermédiarité

Lacunes

Niveau de revendication

Noyau dictatorial

Paires stabilisantes

Part du hasard

Phénomènes paranormaux

Préférences collectives

Probabilité des causes

Procédure de vote

Procédure majoritaire

Règle de choix

Rimes plates et rimes embrassées 